CARNETS OE Carnets de géographes

GÉOGRAPHES.

5 | 2013

Géographie humanimale

\title{
Le protocole du jabiru
}

Agentivités animales et animalières au zoo de Barcelone

Bastien Picard

\section{CpenEdition}

Journals

Édition électronique

URL : http://journals.openedition.org/cdg/1072

DOI : $10.4000 /$ cdg. 1072

ISSN : 2107-7266

Éditeur

UMR 245 - CESSMA

Référence électronique

Bastien Picard, «Le protocole du jabiru », Carnets de géographes [En ligne], 5 | 2013, mis en ligne le 01 janvier 2013, consulté le 30 avril 2019. URL : http://journals.openedition.org/cdg/1072 ; DOI :

$10.4000 /$ cdg. 1072

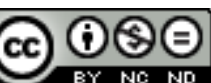

La revue Carnets de géographes est mise à disposition selon les termes de la Licence Creative Commons Attribution - Pas d'Utilisation Commerciale - Pas de Modification 4.0 International. 


\title{
LE PROTOCOLE DU JABIRU
}

Agentivités animales et animalières au zoo de Barcelone

\author{
BASTIEN PICARD \\ Anthropologie Sociale \\ LAS/EHESS \\ bastien.picard@ehess.fr
}

\section{Résumé}

En s'appuyant sur une enquête ethnographique effectuée au zoo de Barcelone ainsi que sur l'éthologie, cet article se propose d'examiner l'articulation entre agentivités animale et animalière au sein des parcs zoologiques. L'analyse des interactions spatiales entre échassiers et animaliers montre que loin d'être toujours passifs, les animaux captifs ont différents moyens d'agir sur leurs soigneurs. D'autre part, ces derniers peuvent choisir d'éviter tout contrôle «direct» et opérer suivant un anthropomorphisme nonanthropocentrique qui les éloigne de l'attitude naturaliste. L'agentivité des animaux captifs s'en trouve renforcée.

\begin{abstract}
Based on an ethnographical survey in Barcelona Zoo and ethology, this article examines how animals' and keepers' agency are attuned in zoos. Analysis of spatial interactions between wading birds and keepers shows that far from being always passive, captive animals display a large panel of actions. Keeper may choose to avoid direct control and act on the ground of a non-anthropocentric anthropomorphism that helps them to move away from a naturalistic attitude, thus strengthening captive animals'agency.
\end{abstract}


Figure 1 : Jabirus d'Afrique (Ephippiorhynchus senegalensis), Zoo de Barcelone

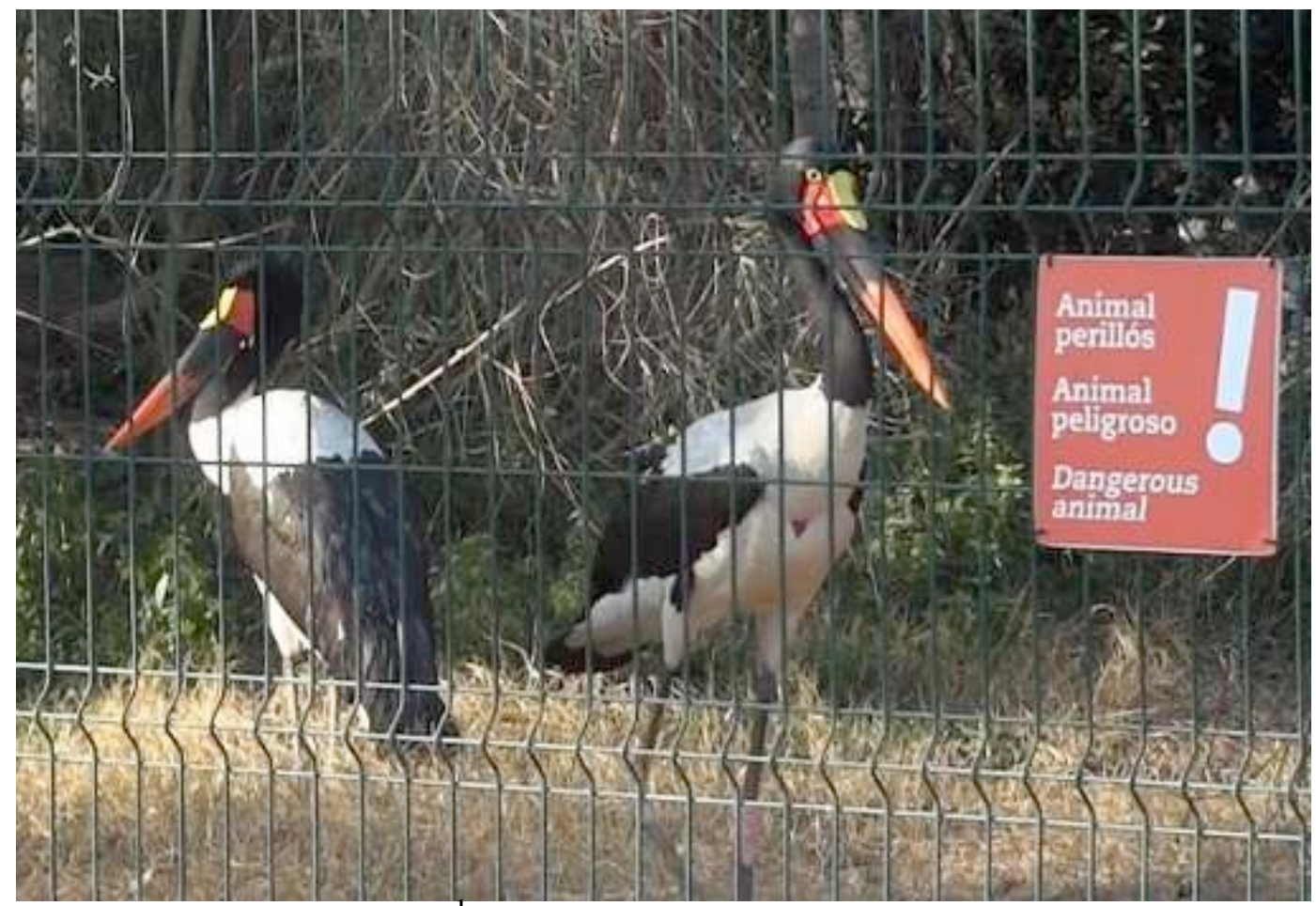

Février 2012, photo B. Picard

\section{Introduction}

Comme le soulignent Mullan \& Marvin (1999: xxi) et Estebanez (2010), les parcs zoologiques et leurs animaliers ont fait l'objet de très peu d'études en sciences humaines et sociales. Pourtant, la captivité des animaux de zoo fait de ce dernier un lieu privilégié pour observer des relations étroites entre des hommes, les soigneurs animaliers, et des animaux présentés comme sauvages. A partir d'une analyse comparative des lieux de confinement, Mullan \& Marvin (1999 : 38-39) caractérisent la relation entre gardiens et captifs par la soumission des seconds aux premiers. Ainsi le «contrôle direct» des soigneurs engendrerait « manque d'initiative » et « docilité » chez les animaux de zoo. Au sein de ces institutions, cette relation de soumission serait considérée comme « à la fois nécessaire et appropriée. » En ce sens l'agentivité serait très inégalement répartie au sein des relations entre animaux et animaliers. A l'agentivité forte voire totale des gardiens répondrait l'« attente passive » (Berger, 1980) des animaux captifs.

Plus largement, l'attitude des animaliers serait la manifestation de la nature anthropocentrique du zoo qui, en dominant des animaux à des fins humaines (Mullan \& Marvin : 160), exprime «la supériorité et la distance [de l'homme] envers le monde naturel » (ibid. : 31). En effet, en tant que lieu de présentation de la « nature », le zoo moderne semble lié à une ontologie particulière, celle que Descola (2005) nomme

\footnotetext{
${ }^{1}$ Il est possible de consulter photographies et vidéos par Josep del Hoyo sur The Internet Bird Collection. http://ibc.lynxeds.com/species/saddlebill-ephippiorhynchus-senegalensis
} 
naturalisme (Servais, 2009 ; Estebanez \& Staszak, 2012). L'ontologie naturaliste est caractérisée par un continuisme des physicalités et un discontinuisme des intériorités (Descola, 2005 : 242-243). Les humains y sont distingués des non-humains par « la conscience réflexive, la subjectivité, le pouvoir de signifier, la maîtrise des symboles », « le langage », ou encore la «culture ». Or, en définissant les non-humains par leur "défaut d'humanité », le naturalisme est la seule ontologie qui soit réellement anthropocentrique (ibid. : 355). En suivant l'analyse de Mullan \& Marvin, on pourrait donc considérer que les relations entre animaux et animaliers ne font qu'exprimer un rapport plus général entre une «nature», objectivée et passive, et une «culture», objectivante, seul véritable lieu de la subjectivité et de l'agentivité. En exerçant un « total contrôle » (Pellegrini, 1995) sur l'animal sauvage, le zoo et son personnel manifesteraient au plus haut point l'attitude anthropocentrique de l'ontologie naturaliste.

Au sens large, l'agentivité peut être définie comme le "pouvoir d'un affectant sur un affecté » (Fontaine, 2010). La biologie phénoménologique et la psychologie écologique ont montré l'existence de l'agentivité animale dans ses relations à l'environnement (Uexküll, 1934/1965 ; Merleau-Ponty, 1995 ; Gibson, 1979 ; Warkentin, 2009). Cet article cherche à préciser les modalités d'action des agents animaux et animaliers lors de leurs interactions, ainsi qu'à examiner la manière dont ces agentivités «s'agencent » (Despret, 2012 : 15). L'analyse portera sur les interactions spatiales entre des soigneurs et une espèce d'échassier : le jabiru d'Afrique.

\section{Éthologie et ethnologie}

Il a été souligné que la présence des non-humains au cœur de la vie sociale des hommes rendait nécessaire une modification des outils de l'anthropologie afin de pouvoir les y intégrer (Descola, 2005: 15 ; Latour, 1991). Afin de ne plus réduire les animaux à une fonction d'entourage, et de permettre leur appréhension comme «agents en interaction avec des hommes dans des situations données » (Descola, 2011: 100), une double approche est devenue nécessaire, mêlant ethnologie et éthologie (ibid. : 102 ; Brunois, 2005 ; Lestel et al., 2006). Cette approche hybride est rendue d'autant plus indispensable dans le zoo qu'il est marqué par une double spécificité.

La première de ces spécificités réside dans la visibilité et la proximité d'animaux dont la principale fonction est d'être exposés. Même si certains enclos sont conçus de telle manière que les animaux puissent se dissimuler, le zoo reste un lieu de clôture et d'exposition qui rend ses animaux presque toujours visibles et proches. C'est aussi vrai des espaces non accessibles aux visiteurs, les «coulisses » où l'observation de l'animal permet de s'assurer de son état de santé ainsi que de la sécurité des lieux. Cette situation singulière rend possible l'observation à faible distance d'interactions rapprochées entre des hommes et des animaux « sauvages ».

La seconde spécificité du zoo qui nous intéresse ici est liée à la temporalité. Les animaliers côtoient quotidiennement et le plus souvent durant des années des animaux dont ils ont une connaissance fine et souvent individualisée. Par ailleurs, avec le temps, 
les animaux de zoo développent aussi certaines connaissances, qu'elles soient liées aux horaires du personnel ou à la reconnaissance individuelle des soigneurs. Enfin, soigneurs et animaux développent parfois des relations affectives qui ne sont pas sans lien avec les savoirs constitués (Despret, 2007 ; Rémy, 2007 ; Estebanez, 2010).

Autrement dit, le caractère étroit des interactions entre animaux de zoo et animaliers est à prendre ici en deux sens : un sens strictement spatial et un sens relationnel et cognitif. Ce caractère étroit a pour conséquence méthodologique de rendre impossible toute occultation du comportement de l'animal de zoo. Afin d'étudier les relations entre animaux et animaliers, il est donc nécessaire d'allier science de l'homme et science de l'animal, ici ethnologie et éthologie. Il ne s'agit pas de considérer l'éthologie comme une voie d'accès objective au comportement animal qui permettrait de décider de la valeur de vérité des savoirs animaliers. Il s'agit plutôt d'utiliser un outil supplémentaire pour comprendre des interactions dans lesquelles le comportement animal est producteur d'effets. Par sa perception détaillée des comportements, l'éthologie permet de rendre manifestes les manières d'agir animales. Cependant, la frontière entre éthologie et ethnologie devient parfois ténue lorsqu'il s'agit d'observer des interactions dans lesquelles la communication, y compris humaine, est non-verbale (Joulian, 1999; Leblan, 2011 ; Servais \& Servais, 2009), s'effectuant « de corps à corps »(Vialles, 2004).

Cet article s'appuie sur une enquête ethnographique effectuée au zoo de Barcelone de juillet 2011 à mars 2012. L'essentiel du travail de terrain a consisté à suivre plusieurs animaliers durant leur journée de travail. Les informations ont été collectées à partir d'observations notées par écrit et de discussions informelles qui se sont déroulées durant le temps de travail ou peu de temps après. Durant les interactions entre animaliers et jabirus d'Afrique, la méthode d'observation a finalement été la même pour l'homme comme pour l'animal. Elle a consisté à noter chaque action, geste, déplacement ou position des protagonistes. Bien que se focalisant systématiquement sur la dyade en interaction, ce type de notes s'apparente à la méthode d'échantillonnage ad libitum (ad libitum sampling) (Altmann, 1974), dans la mesure où le flux comportemental n'a pas été segmenté en unités de base visant une étude quantitative. Ce mode de description qualitatif des comportements a été utilisé dans les travaux éthologiques les plus proches de l'ethnographie (Goodall, 1986).

Malgré son manque de systématicité d'un point de vue quantitatif, ce type de description reste adapté lorsqu'il porte sur des interactions sociales (Nakamura, 2009), en particulier si le nombre de protagonistes est réduit. D'abord parce que l'interaction, en tant qu'action réciproque, ne peut se décomposer en actions alternées, ce qui rend la catégorisation des actions plus difficile. Ensuite, parce qu'une interaction sociale changeant de manière dynamique, il est nécessaire d'éviter tout schématisme rigide. Lors de l'observation, la description qualitative permet d'approcher cette dynamique en notant le détail des interactions, bien qu'elle soit déjà une manière de figer le flux comportemental en en extrayant des fragments. 


\section{Situation d'interaction}

Fondé en 1892, le Parc Zoologique de Barcelone est un zoo municipal situé au cœur de la ville. Recensant 2208 animaux et 318 espèces pour 13 hectares, c'est un zoo à forte densité animale. Le personnel animalier y est réparti en cinq sections: Primates, Mammifères marins, Collection générale (mammifères autres que primates et mammifères marins), Terrarium, et Aviaire. Chaque section est divisée en un certain nombre de services qui consistent à faire la tournée de plusieurs installations afin de les nettoyer et de nourrir les animaux. Durant la matinée (7h30-14h30), la section Aviaire comporte quatre services. Deux d'entre eux se consacrent aux animaux situés à l'intérieur des bâtiments, les deux autres se partageant les oiseaux exposés à l'extérieur. Le jabiru d'Afrique, auquel nous allons ici nous intéresser principalement, appartient à l'un de ces deux derniers services.

Le jabiru d'Afrique (Ephippiorhynchus senegalensis) est un échassier de la famille des Ciconiidés. Mesurant de 1,45 $\mathrm{m}$ à 1,50 $\mathrm{m}$ de hauteur pour une envergure de 2,40 $\mathrm{m}$ à $2,70 \mathrm{~m}$, il pèse environ $5 \mathrm{~kg}$. Habituellement solitaire ou en couple, il forme parfois des groupes comprenant jusqu'à une douzaine d'individus (Hoyo et al., 1992: 463). La volière des jabirus du zoo de Barcelone est occupée par un couple, cohabitant avec un petit groupe d'ibis sacrés (Threskiornis aethiopicus) ; elle consiste en un enclos grillagé de forme rectangulaire, dont le sol herbeux est ponctué de quelques arbustes et de deux plates-formes.

Les animaliers que nous avons pu observer ont des profils contrastés. La première, E., est une jeune soigneuse qui effectue des remplacements dans la section Aviaire depuis deux ans. Elle a par ailleurs une formation en biologie. Le second soigneur, D., travaille depuis quarante-cinq ans au zoo de Barcelone, dont trente années comme titulaire du service qui comprend aujourd'hui les jabirus d'Afrique. On nous a rapporté par ailleurs les manières d'agir d'autres animaliers non identifiés.

Les interactions entre animaliers et jabirus ont été observées durant les mois de juillet et décembre 2011, ainsi que durant le mois de janvier 2012. Lors de la matinée (7h3014h30), les situations d'interaction sont au nombre de deux ou trois selon les journées. Elles coïncident avec l'entrée de l'animalier dans la volière afin d'y déposer de la nourriture. La durée totale de l'intervention du soigneur se situe entre cinq et dix minutes, avec une moyenne de huit minutes par intervention. La nourriture (viande hachée, vers de farine) est généralement déposée dans des bacs placés sur le sol à l'entrée de la volière. Cependant, certains jours l'animalier doit atteindre le centre de l'installation afin de déposer des anguilles, vivantes, dans un petit bassin. Pour cela, il doit suivre un chemin détourné car la zone centrale étant occupée par le couple de jabirus, celle-ci n'est pas approchée. L'animalier qui entre dans la volière doit donc en longer le bord sud et passer à la gauche du nid, pour ensuite revenir vers le centre et le bassin. La difficulté réside dans l'attitude parfois agressive du mâle jabiru, y compris en dehors des périodes de nidification. En effet, si la femelle se contente d'observer la progression du soigneur depuis l'espace central, le mâle, lui, le suit à distance, l'approche, jusqu'à parfois le menacer. Or, le long bec aigu du jabiru d'Afrique peut constituer un risque tout à fait 
sérieux. Malgré l'incrédulité des visiteurs devant la pancarte "animal dangereux» apposée à la volière, le péril existe bien. L'EAZA, l'association européenne des zoos et aquariums, classe d'ailleurs Ephippiorhynchus senegalensis parmi les animaux dangereux (EAZA, 2008).

L'agent animal principal des interactions qui nous occupent ici est donc le mâle jabiru. Cette focalisation est moins l'effet d'une moindre attention portée à la femelle (Despret, 2002: 169) que la conséquence du moindre impact de son comportement sur le déroulement des interactions avec les animaliers. En effet, si la femelle est parfois la plus active (notamment lorsqu'il s'agit de chasser les ibis), durant l'entrée du soigneur son unique action consiste à se tenir immobile au centre de la volière, et à l'observer à distance. Sa présence a probablement plusieurs effets, dont la défense du centre de la volière par son partenaire et son évitement par l'animalier. Cependant, le soigneur interagit principalement avec le mâle, celui-ci accaparant toute son attention une fois la position de la femelle identifiée. De même, s'il est fait très peu mention des oiseaux qui partagent la volière avec les jabirus, ce n'est évidemment pas pour nier qu'ils puissent être des agents, mais parce que leur présence n'induit pas d'interactions. Perchés sur les arbustes ou les plates-formes, les ibis attendent que la nourriture soit déposée, et l'animalier semble les ignorer totalement lorsqu'il entre dans la volière. De manière générale, les animaliers semblent n'avoir qu'un faible intérêt pour les ibis, ceux-ci n'étant que très rarement mentionnés dans les conversations portant sur les oiseaux de l'installation.

\section{Actions du mâle jabiru : observation et agression}

Face à l'animalier, le mâle jabiru peut adopter deux types de comportement. D'abord un comportement d'observation, mobile ou immobile. Si le soigneur vient du sud et longe la volière de l'extérieur, le mâle le suit en marchant à sa hauteur. Lorsque l'animalier arrive à la porte d'entrée de l'installation, le jabiru le suit du regard et s'approche. Une fois le soigneur entré, l'oiseau entame un suivi à distance en empruntant un chemin parallèle passant par le centre de l'installation. Lorsque l'animalier atteint le bassin, le jabiru s'arrête généralement à quelques mètres pour l'observer. L'animal peut également adopter un comportement d'agression. Ce dernier peut être défini comme un comportement relationnel ayant « pour fonction la mise à distance ou le maintien d'une distance entre des individus appartenant ou non à la même espèce » (Deputte, 2007). Dans ce cas, le jabiru commence généralement par s'approcher beaucoup plus près, ce qui suffit parfois à faire reculer l'animalier. Si ce dernier ne fuit pas, l'oiseau entame une démonstration (display). Il se positionne face à sa cible et tente de l'intimider en déployant ses ailes. Comme le souligne E. : « Ça signifie: "Regarde comme je suis grand !" ». Un troisième type de comportement agressif consiste en un claquement de bec avec mouvement de ce dernier dirigé vers la cible. Ce mouvement d'attaque incomplet correspond à un «mouvement d'intention» (ibid.), une ébauche de comportement à but communicationnel. L'ensemble de ces manifestations d'agressivité constitue des « signaux de communication permettant de moduler l'expression [de la] motivation [agressive] en fonction de la réponse » du protagoniste (ibid.). Les interactions entre jabirus et 
animaliers sont donc sociales, au moins en un sens minimal. D'abord parce que ces relations impliquent une certaine réciprocité. « Il y a société là où il y a action réciproque de plusieurs individus » (Simmel, 1908/1999 : 43 ; Goldberg, 1998 : 32). Ensuite parce qu'elles supposent la communication (Bateson, 1936/1986: 221 ; Servais \& Servais, 2009).

Deputte souligne que «les comportements d'agression s'inscrivent [...] fondamentalement dans une relation triangulaire, l'individu, le protagoniste, et la "ressource" », celle-ci pouvant être « d'ordre alimentaire, spatial (la compétition pour un site de sommeil ou de nidification), ou sexuel ». De fait, pour les animaliers, le mâle jabiru marque par là son territoire. Ils font ainsi écho à la définition éthologique la plus commune du territoire comme «aire défendue » (Maher \& Lott, 1995). Cette fonction territoriale des communications est d'ailleurs souvent mentionnée par les soigneurs, qu'il s'agisse d'une territorialité intraspécifique ou interspécifique. Selon les animaliers, l'agressivité du jabiru est donc distincte de celle des oiseaux imprégnés, qui n'ont aucune peur de l'homme et n'hésitent pas à entrer en contact avec lui. L'échassier, au contraire, évite toujours le contact physique. Il menace à distance. L'éthologie en parcs zoologiques fait écho au point de vue des animaliers en soulignant que «l'animal en captivité est enclin à considérer l'espace qui lui est assigné comme son territoire personnel » (Hediger, 1953 : 99; Goldberg, 1998: 143). L'espace joue donc un rôle essentiel dans les interactions entre échassiers et animaliers. En constituant la volière comme son territoire, le comportement du jabiru va directement agir sur la manière dont vont répondre les animaliers.

\section{Actions animalières : annonce, observation et protection}

Annonce. L'interaction avec les jabirus commence dès le premier contact visuel, avant même que l'animalier soit entré dans la volière. Le premier type d'action consiste à s'annoncer pour ne pas effrayer les oiseaux en les surprenant. Des sifflements préventifs sont les plus courants et sont fréquemment utilisés par les animaliers de toutes les sections du zoo. D. a aussi développé des actions qui lui sont propres. Il choisit par exemple d'effectuer des détours lors de sa ronde afin d'arriver par le côté où l'animal a la vue la plus dégagée. L'hiver, il attend que le jour soit suffisamment levé pour se présenter devant la volière. Une fois devant, il effectue des gestes lents afin de permettre à l'oiseau d'anticiper son entrée.

Observation. Que ce soit à l'intérieur ou à l'extérieur de la volière, l'animalier ne cesse d'observer les indices corporels du jabiru afin d'anticiper une agression. Aux expressions comportementales déjà mentionnées, il faut ajouter une particularité anatomique d'Ephippiorhynchus senegalensis : le poitrail de l'échassier présente une petite zone de peau rouge dénuée de plumes dont la couleur s'intensifie plus ou moins en fonction de son état d'excitation (sexuelle ou agressive). Le soigneur peut ainsi mesurer son niveau d'agressivité, ou de « colère », de façon permanente. 
Protection passive. Cette protection peut être continue, comme un balai maintenu au niveau du visage ou un col relevé, ou ponctuelle, par exemple en se positionnant de dos lorsque le jabiru s'approche.

Protection active interruptive. Il s'agit de se protéger en accomplissant une action visant à désamorcer l'agression. Il est possible que le positionnement de dos fasse partie de ce type de protection. En effet, bien que le contexte soit interspécifique, on peut penser que la position du soigneur agisse comme un signal d'interruption (cut-off signal) : «Le regard, la face et/ou l'ensemble de la posture sont détournés de leur direction vers le protagoniste. L'expression même de l'agression, orientation vers l'adversaire et préparation à l'attaque, se trouve donc éliminée, abaissant ainsi la probabilité de la poursuite du conflit. » (Deputte, 2007). On observe que l'oiseau stoppe souvent son approche lorsque l'animalier se détourne pour lui présenter son dos. Ce positionnement semble donc agir de deux manières : comme signal d'interruption (pour l'animal), et donc comme protection (pour l'animalier). Lors d'une conversation avec E., D. mentionne un autre moyen de protection interruptive : «Quand je versais les anguilles, je tenais les anguilles de la main droite et le seau de la main gauche, de son côté. Comme ça, s'il s'approchait, je levais légèrement le seau. Pas le rapprocher de lui, mais le lever un peu, de manière que cela le surprenne. » Un autre moyen pour interrompre une approche et conserver une distance de sécurité est de surprendre l'animal. Ce qui nous intéresse ici, c'est que la réduction de la distance entre l'animal et l'animalier n'est pas envisagée. Il ne s'agit pas d'avancer vers l'animal, ce qui signifierait un affrontement, mais de « lever un peu » le seau pour le surprendre. Il ne s'agit pas non plus de le faire reculer ou de l'éloigner, mais de le maintenir à une distance minimale. Désarçonner plutôt qu'affronter. Nous verrons qu'il s'agit là d'un choix entre deux attitudes possibles envers les animaux de zoo.

Protection spatiale. Les moyens de protection précédents avaient rapport à l'espace, mais il s'agit ici d'un type de protection entièrement tourné vers la gestion de zones ayant des significations différenciées. Ainsi D. conseille à E. de disposer les bacs destinés à la nourriture " plus à l'intérieur de l'installation », pour deux raisons : d'abord parce que c'est «plus dans son espace », ce qui permet à l'échassier de manger sans être inquiété par les déplacements du soigneur. Pour D., la zone la plus intérieure de l'installation appartient au jabiru. C'est pourquoi l'animalier doit effectuer un détour pour atteindre le bassin. L'utilisation du comparatif «plus» signifie aussi que la différenciation spatiale de la volière s'opère graduellement à partir d'un centre d'appropriation, et donc d'exclusion des intrus, le cœur du territoire jabiru, jusqu'à une périphérie accessible sans danger aux animaliers. La seconde raison, c'est que «ça laisse plus d'espace » à l'animalier pour effectuer sa sortie.

\section{Configuration spatiale des interactions entre jabirus et animaliers}

A partir des éléments précédents, nous pouvons commencer à dessiner la configuration spatiale de la situation décrite. Nous mettons de côté l'espace aérien, réservé aux ibis, car 
il n'a pas d'effet observable sur les interactions entre jabirus et animaliers : nous avons déjà mentionné que les ibis restaient immobiles durant toute l'intervention du soigneur.

Un premier espace, situé au centre de la volière et incluant le nid, correspond au cœur de l'espace propre des jabirus. Cette zone a toujours été contournée par les animaliers. C'est aussi le lieu d'observation de la femelle. Un second espace est constitué par la périphérie du territoire jabiru, qui comprend la zone intérieure à proximité de la porte d'entrée ainsi que le chemin qui contourne le premier espace jusqu'au bassin. C'est le seul espace situé à l'intérieur de la volière qui soit parcouru par les animaliers. C'est aussi là qu'ont lieu les démonstrations d'agressivité. Un troisième espace correspond à l'entourage extérieur immédiat de la porte d'entrée. L'animalier doit retirer doucement le cadenas de la porte tout en évaluant l'attitude du jabiru, qui peut tenter de donner des coups de bec à travers la grille; c'est donc la première zone de contact potentiel entre l'animal et l'animalier. Un quatrième espace se situe autour de la barrière empêchant les visiteurs de s'approcher de la volière. C'est là que $\mathrm{D}$. commence à préparer lentement son entrée. Le jabiru, immobile, l'observe depuis la zone intérieure située près de la porte d'entrée. Enfin, un cinquième espace est constitué par le chemin emprunté par les visiteurs et l'animalier. Lorsque ce dernier arrive devant la volière, il est déjà observé et suivi par l'échassier.

Autrement dit, l'espace intérieur de la volière n'est pas homogène. Plus précisément, et comme nous le soulignions plus haut, l'intérieur de l'installation s'organise à partir d'un centre appartenant au couple de jabirus (espace 1), centre qui irradie en s'affaiblissant jusqu'à une périphérie accessible aux animaliers (espace 2). Que ce soit du point de vue animal ou du point de vue animalier, on a donc affaire à une différenciation graduelle de l'espace, et non à une juxtaposition de zones dont les frontières seraient aisément déterminables. C'est pourquoi le soigneur peut entrer plus avant dans l'espace du jabiru pour y déposer la nourriture. Cependant, bien que les limites ne soient pas invariablement fixées, il existe toujours une zone inaccessible pour l'animalier.

Il faut souligner que cette différenciation graduelle de l'espace s'étend au-delà de la volière. En effet, l'espace 3 s'apparente à l'espace 2 en ce qu'il peut déjà y avoir contact et agression. D'autre part, l'espace 4 s'apparente aux espaces 2 et 3 en ce qu'il y a déjà interaction entre le jabiru et l'animalier. Ce dernier se sait observé et prépare, par ses gestes lents, son entrée dans la volière. Enfin, l'espace 5 est déjà le lieu d'un contact visuel et d'une anticipation de la part des protagonistes. Cela signifie que la distinction intérieur/extérieur ne suffit pas pour rendre compte des interactions que nous avons décrites. Certes, de manière évidente, l'animal ne peut sortir de l'installation par luimême, ce qui suffirait à reconnaître l'existence d'une limite à la fois stable et nettement dessinée, limite qui correspondrait à la clôture. Pourtant, dans les interactions observées, on ne peut distinguer un espace intérieur, animal, et un espace extérieur, humain, comme s'ils étaient homogènes et clairement séparables l'un de l'autre. Du point de vue des animaux de zoo et des animaliers, l'espace est beaucoup plus différencié.

La configuration que nous venons de décrire est restée relativement stable au cours de nos observations. La présence du public, par exemple, n'a pas eu de réel impact. De même, entre l'été 2011 et l'hiver 2011-2012, nous n'avons pas observé de modifications 
majeures en dépit des variations de l'environnement (température, heure du lever du soleil). Nous n'avons malheureusement pas pu observer ces interactions dans des conditions météorologiques variées (pluie, vent), ces dernières ayant été clémentes durant chaque session d'observation. Si le cadre spatial est resté relativement stable, les interactions qui se sont déroulées au sein de ce cadre ont néanmoins fluctué sous l'effet de plusieurs facteurs.

D'abord, la disposition du mâle jabiru semble jouer un rôle important. Pour les soigneurs, son comportement est sujet à des variations dont la cause souvent leur échappe. «On ne sait pas pourquoi aujourd'hui il est plus agressif. » Si la faible fréquence des entrées dans la volière semble expliquer un fort niveau d'agressivité, l'oiseau déçoit régulièrement les attentes, y compris parfois par sa faible animosité. Loin de réagir de manière identique aux attitudes des animaliers, le jabiru peut ainsi créer la surprise. Comme le souligne D., «c'est pas automatique ».

Ensuite, l'identité de l'animalier joue peut-être un rôle. En effet, certains oiseaux du zoo reconnaissent les soigneurs de manière individuelle et on observe que E. a beaucoup plus de difficultés que $\mathrm{D}$. pour gérer l'agressivité du jabiru. L'individualité de l'animalier perçue par le jabiru est probablement liée à sa démarche, à ses gestes (assurés ou craintifs), ou à sa vitesse. Cependant, dans la mesure où l'individualité de l'animalier est fortement liée à sa manière d'agir, à son «style ", il est difficile de la séparer du type d'approche adopté. Il convient donc de se pencher plus en détail sur ces approches animalières.

\section{« C'est comme un protocole. »}

D. décrit les différentes activités assurant le bon déroulement de ses interactions avec le jabiru (lenteur des gestes, laisser du temps, ...) d'une manière toute particulière. Dans une conversation avec E., il répète deux fois que pour lui : "c'est comme un protocole. » «Comme un protocole», ça signifie que ça y ressemble sans y être identique. Examinons le sens de cette comparaison.

Dans un premier sens, un protocole est une convention, ou le contenu de cette convention. Dans cette acception, un protocole nécessite une délibération entre au moins deux parties, délibération aboutissant à l'adoption de résolutions. Rien ne venant supporter l'idée d'une délibération entre jabirus et animaliers, il convient d'écarter cette acception du terme.

Dans un second sens, un protocole est une prescription précise des conditions et du déroulement d'une opération. Suivre le protocole, c'est s'assurer de répéter l'opération dans les meilleures conditions en reproduisant chacune de ses étapes à l'identique. En ce sens, D. souligne l'efficacité de la reproduction des mêmes gestes : « Je ne sais pas si c'est pour ça, mais ça fonctionne pour moi. » Au scepticisme relatif à la nature des causes qui sont en jeu répond la certitude de l'efficacité du protocole. Le protocole a une valeur pragmatique. En outre, le fait qu'il fonctionne montre qu'il entre en cohérence avec 
ce que l'animalier sait et perçoit de l'animal, et avec ce que l'animal, de son côté, semble percevoir de l'animalier, mais sans la certitude que cette cohérence s'ancre dans la réalité de causes sous-jacentes. Le protocole pourrait ici être dit «phénoméniste $»^{2}$.

Enfin, dans une troisième acception, un protocole c'est d'abord un recueil de règles à observer en matière d'étiquette, de préséances, dans les cérémonies et les relations officielles. Il s'apparente alors à un cérémonial, à l'énoncé des formes imposées dans la vie en société. Cette acception sociale du protocole ne peut être écartée. Les animaliers ont bien conscience d'établir une relation sociale avec un animal qui se comporte selon certains usages. Par ailleurs, D. emploie ce terme dans un contexte qui suggère qu'il l'utilise aussi dans son acception sociale. Alors que nous sommes en train d'observer la parade nuptiale d'un groupe de flamants de Cuba (Phoenicopterus ruber), il commente: « Tu vois comme ils baissent le cou, comme s'ils allaient s'arranger les plumes. Mais ils ne le font pas. Disons que ça fait partie du protocole de la danse. » Si D. compare les différentes étapes assurant le bon fonctionnement de ses interactions avec le jabiru à un protocole, c'est donc aussi parce qu'il est nécessaire de suivre des règles qui ont une signification sociale pour l'animal. Lorsqu'il pénètre dans son territoire, D. suit certains de ces usages : il s'annonce et évolue lentement. S'il doit se protéger, il le fait en se positionnant de dos, ou en levant légèrement le seau, et non pas en le rapprochant, ce qui pourrait apparaître comme une manifestation d'hostilité. Même le retrait, ultime recours, est adapté et réglé, puisqu'il s'effectue lui aussi de manière lente, afin d'éviter d'apparaître comme une « proie » facile et de subir une agression.

Heini Hediger, alors directeur du zoo de Zurich, rappellait que le dresseur doit se conformer au "cérémonial» de l'espèce (Hediger, 1953 : 215). Ainsi le dompteur de grands carnivores doit comprendre le fonctionnement de la hiérarchie sociale des espèces auxquelles il est confronté afin d'utiliser ces règles sociales à son avantage. Il doit notamment se maintenir au sommet de la hiérarchie interspécifique en assurant sa domination territoriale (ibid. : 64). Pour Hediger, la situation du dresseur est telle parce que l'animal dressé le considère et le traite comme un membre de son espèce. Si ce type de zoomorphisme existe chez les oiseaux et peut être utilisé (Pellegrini 1995), il n'y a ici aucune évidence de ce que le jabiru considère les animaliers comme des membres de sa propre espèce. S'il les intègre dans son "monde » social, il est peu probable qu'il tolère qu'un membre de son espèce entre dans son territoire trois fois par jour. Il est possible d'entretenir des relations sociales interspécifiques et d'accepter le «cérémonial»de l'espèce sans être nécessairement considéré par l'animal comme un membre de sa propre espèce.

Pour D. donc, si sa manière d'interagir avec le jabiru est « comme un protocole », c'est d'abord parce qu'elle assure une efficacité relationnelle minimale. Mais c'est aussi parce qu'elle ressemble au respect des formes dans la vie en société. De même que le dresseur peut suivre le « cérémonial» de l'espèce, et l'éthologue ses «bonnes manières » (Joulian,

2 Ce terme est ici utilisé en référence au positionnement philosophique couramment appelé « phénoménisme» (Protagoras, Sextus Empiricus, Pierre Duhem). Selon celui-ci, connaitre, c'est « sauver les apparences ». La connaissance se construit en accord avec les phénomènes observables, sans se demander si cette connaissance atteint le cœur du réel. 
1999) ou ses « bons usages » (Despret, 2012 : 178), le soigneur peut suivre le protocole, c'est-à-dire les usages sociaux de l'animal avec lequel il interagit. Si néanmoins cette approche est « comme un protocole », c'est que la sociabilité interspécifique n'implique pas le même type d'engagement que la sociabilité interhumaine (Vialles, 2004). On peut donc établir une première distinction chez les animaliers selon que leur approche est plus ou moins protocolaire. Si E. essuie plus d'échecs que D., c'est parce qu'elle manque d'expérience et maîtrise moins les usages jabirus. Bien qu'imparfaite, son approche reste néanmoins protocolaire dans sa visée.

\section{Approche oblique et approche en face-à-face}

$\mathrm{Si}$ D. et le dresseur décrit par Hediger ont en commun d'adopter une approche protocolaire, leur mode d'acceptation du «cérémonial» de l'espèce diffère. Alors que le dompteur accepte un minimum de règles afin de s'assurer une position de domination, $\mathrm{D}$. évite soigneusement le conflit et rebrousse chemin si la situation s'envenime. Cette différence pourrait s'expliquer par le fait que, à la différence de la situation du dompteur, l'animal est ici le premier occupant de l'espace d'interaction: l'animalier entre dans une volière que l'oiseau ne quitte jamais. Elle pourrait aussi s'expliquer par la nature des espèces (Hediger, 1953 : 66). Pourtant, la situation n'impose aucun déterminisme et l'attitude de D. n'est pas la seule possible. En effet, pour E., « il y a deux théories ». Face à une menace du jabiru, «D. est partisan [...] de revenir plus tard. L'autre théorie, c'est de faire face car sinon c'est lui qui a gagné. »

Comme le suggère E., on peut distinguer deux "théories », ou deux approches : une approche en face-à-face, frontale, et une approche oblique. Selon la première, les animaliers choisissent d'affronter le jabiru afin de ne pas lui laisser l'exclusivité de l'espace constitué par la volière. On retrouve là une attitude plus proche de celle du dompteur décrit par Hediger. L'idée, c'est que battre en retraite renforce l'échassier dans son agressivité.. Selon la seconde approche, au contraire, si on fait face, «ça peut être une boucle. Car il peut aussi devenir plus agressif.» «On ne sait pas pourquoi aujourd'hui il est plus agressif. C'est mieux de revenir plus tard. » (E.) Cette approche oblique est aussi utilisée par D. pour réduire l'agressivité des manchots de Humboldt (Spheniscus humboldti) envers les animaliers, agressivité liée à la «défense du territoire ». Pour cela, D. enjambe la barrière de l'installation afin d'entrer dans l'espace des animaux. Puis, il pose le seau contenant la nourriture, s'agenouille, et saisit deux ou trois manchots, toujours les mêmes, qu'il place autour de lui. L'autre manière de nourrir les manchots consiste à le faire de face. L'animalier reste sur le bord du bassin, seul, et les oiseaux sont disposés face à lui. D. choisit au contraire de s'entourer des animaux et, s'il choisit toujours les mêmes, c'est que pour lui « ils aiment bien monter ». Autrement dit, dans ce type d'interaction, il n'y a pas d'affrontement. L'agressivité potentielle est bien présente, puisqu'il s'agit de la baisser. Mais par le biais de l'habituation, on agit sur elle sans lui faire face.

Or, dans les situations décrites ici, cette différence d'approche est exprimée spatialement. Lorsque D. nourrit les manchots, il en a certes en face de lui, mais il en a 
aussi qui sont disposés à ses côtés. Dans la volière des jabirus, si l'échassier fait mine de s'approcher, il le surprend en levant un peu son seau, mais évite de le rapprocher davantage. Si l'oiseau est déjà proche, il se positionne de dos, mais légèrement de biais, afin de pouvoir l'observer. En dernier recours, il bat en retraite. Au contraire, si l'animalier choisit l'approche frontale, il doit faire reculer l'échassier et avancer sur lui. Il $\mathrm{y}$ a donc ici face-à-face en un double sens : un sens spatial (animaux et animaliers sont l'un devant l'autre, chacun vis-à-vis de l'autre) et un sens agonistique (l'affrontement).

La différence entre l'approche en face-à-face et l'approche oblique repose sur une différence de hiérarchisation des volontés. La première privilégie la volonté des hommes. En effet, si le dresseur épouse certains usages de l'animal, c'est pour pouvoir diriger l'interaction. Les soigneurs-entraîneurs d'éléphants (Loxodonta africana) et de dauphins (Tursiops truncatus) que nous avons rencontrés confirment cette idée. S'ils distinguent des actions «volontaires » chez leurs animaux, ces volontés doivent néanmoins être subordonnées. D'autre part, s'ils reconnaissent des styles d'entraînement au contrôle plus ou moins important (Servais \& Servais, 2009), à la fin « il faut qu'on ait toujours le contrôle, qu'ils fassent ce qu'on demande. » De même, l'animalier qui choisit d'affronter le jabiru cherche à imposer sa volonté en décidant de ne pas revenir plus tard. Au contraire, l'approche oblique privilégie les volontés animales. Critiquant des ornithologues bredouilles venus capturer des oiseaux non-captifs, D. relève qu'ils se sont trompés en croyant que les oiseaux allaient venir à l'heure choisie sans avoir pris en compte leur point de vue. Il souligne alors une caractéristique commune au traitement des oiseaux captifs et non-captifs. «Ils [les ornithologues] disent : "quand moi je veux." Mais c'est pas comme ça. C'est quand les animaux veulent. [...] C'est pas automatique. » D. décrit les volontés animales comme des volontés d'être à une certaine place à un certain moment. C'est quand ils veulent que les goélands descendent, et c'est quand il veut que le mâle jabiru accepte la présence de l'animalier sur son territoire. Et si l'échassier ne veut pas, comme le rappelle E., il faut « revenir plus tard».

On peut donc établir une seconde distinction au sein des approches animalières en fonction de leur caractère frontal ou oblique. On peut alors la combiner avec la première et distinguer plusieurs profils. L'animalier qui affronte le jabiru a une approche nonprotocolaire et frontale. Il ne s'adapte pas aux usages de l'animal et impose sa volonté. Le dresseur a une approche protocolaire et frontale. S'il adopte certains usages, c'est pour mieux subordonner la volonté animale à la sienne. E. a une approche imparfaitement protocolaire et oblique. Enfin, D. a une approche protocolaire et oblique. Il suit les usages et les volontés animales. Ce profil reste néanmoins général dans la mesure où les circonstances peuvent imposer un certain type d'approche plutôt qu'un autre. C'est le cas de la capture, qui consiste à plier la volonté des animaux aux exigences spatiales et temporelles des hommes. Les animaux doivent se situer à tel endroit (la cage, la clinique) au moment où les hommes (les vétérinaires, les animaliers) le veulent. Le zoo reste un espace de confinement organisé par les hommes.

Cela montre cependant que là où il y a une marge de manœuvre, les animaliers peuvent choisir de ne pas exercer un «contrôle direct» sur les animaux. Carole Ferret (2012) distingue les actions directes, qui conduisent sans détour à leur objectif, et les actions 
indirectes, qui atteignent leur objectif par une voie détournée. L'approche protocolaire est indirecte en ce qu'elle passe par l'acceptation de certains usages animaux pour atteindre son objectif. L'approche que nous avons qualifiée d'oblique est également indirecte en ce qu'elle passe par l'adaptation aux volontés animales, et non par l'accomplissement immédiat des volontés humaines. En ce sens, cette dernière approche peut être qualifiée de participative. «L'action sera dite participative si l'objet [de l'action] participe activement à l'action et s'il aurait pu ne pas y participer. » (Ferret, 2006 : 44). Si Ferret retient le critère de la non nécessité afin d'« éviter d'avoir à parler d'intention chez les animaux » (ibid. : 43), en utilisant le vocabulaire des animaliers on pourrait dire que l'action participative requiert la collaboration des volontés animales. Ce qui se vérifie a contrario lorsque l'action échoue du fait du refus de l'animal. En ce qui concerne le jabiru, ses actions sont directes lorsqu'il agresse l'animalier, et passives lorsqu'il le laisse entrer. À la différence de l'action active qui consiste à « faire », l'action passive consiste à « ne pas faire », à laisser faire (ibid. : 26). Il est difficile de savoir si cette action passive est aussi participative dans la mesure où l'objectif du jabiru nous reste inconnu. Ce serait le cas si l'objectif de son action passive était le dépôt de nourriture au centre de la volière. On voit donc que le lieu constitué par le parc zoologique ne détermine totalement ni les attitudes animalières ni les attitudes animales (Rémy, 2007). La situation de captivité ne condamne pas les animaliers au contrôle direct ou total, comme elle ne condamne pas les animaux à la passivité : " s'adapter aux animaux, c'est accepter de ne pas avoir d'eux une maîtrise absolue et immédiate»" (Porcher, 2003).

\section{Anthropocentrismes et anthropomorphismes}

Si l'approche en face-à-face est anthropocentrique, les approches protocolaire et oblique peuvent être qualifiées de zoocentriques. En ce sens, l'attitude du dresseur occupe une position intermédiaire entre l'animalier frontal et les animaliers D. et E.. Il combine en effet le zoocentrisme de l'adaptation aux usages animaux et l'anthropocentrisme lié au privilège de sa volonté. Quant à E. et D., leur approche est doublement zoocentrique puisqu'elle vise à s'adapter au protocole et à la volonté des animaux avec lesquels ils interagissent. L'attitude des animaliers peut donc échapper dans une certaine mesure à l'anthropocentrisme. On peut en effet distinguer deux types d'anthropocentrismes. Le premier désigne le fait que l'homme pense et perçoit depuis une perspective humaine. Malgré le caractère en un sens indépassable de cet "anthropocentrisme incarné » (Karlsson, 2012), les approches protocolaire et oblique sont relativement peu anthropocentrées. Le second type d'anthropocentrisme consiste à positionner l'homme au sommet d'une hiérarchie axiologique. En ce sens, l'approche oblique n'est pas anthropocentrique puisqu'elle subordonne la volonté humaine à la volonté animale.

En attribuant protocoles et volontés aux animaux, les animaliers font preuve d'une certaine forme d'anthropomorphisme, non pas au sens restreint d'une projection, nécessairement erronée, de caractéristiques propres à l'homme sur des non-humains, mais au sens large d'une attribution de propriétés humaines à des non-humains. Cet anthropomorphisme zoocentrique correspond à ce que de Waal (1999) nomme 
anthropomorphisme animalocentrique (animalcentric). Celui-ci cherche à comprendre, autant que possible, les animaux dans leurs propres perspectives. Il est donc empathique au sens où il cherche à se mettre à la place de l'autre. Il nécessite pour cela certaines connaissances des habitudes de l'espèce voire des individus. Tout en reconnaissant la différence des perspectives animales, il se distingue de l'«anthropodéni » (anthropodenial) en ce qu'il ne nie pas l'existence chez les animaux de caractéristiques similaires à celles de l'homme. «Nous sommes parfaitement capables de dresser des parallèles avec notre propre expérience sans nier de possibles différences. [...] Lorsqu'une soigneuse animalière dit "délicieux" alors qu'elle nourrit un singe-écureuil avec des vers de farine, elle parle pour l'animal, non pour elle-même. » (ibid.)

De Waal distingue cet anthropomorphisme de l'anthropomorphisme anthropocentrique. Celui-ci consiste à projeter des caractéristiques humaines sur les animaux sans s'appuyer sur aucune connaissance. Lorqu'elle privilégie les besoins des visiteurs et du personnel au détriment de ceux des animaux, la conception des installations zoologiques manifeste ce type d'anthropomorphisme sous la forme d'un "anthropomorphisme par omission », c'est-à-dire une « incapacité à considérer que les animaux ont des mondes différents du nôtre » (Rivas \& Burghardt, 2002). C'est ce même type d'anthropomorphisme qui consiste à affirmer la supériorité de l'homme du fait de l'absence d'une caractéristique proprement humaine chez l'animal. L'anthropomorphisme anthropocentrique est aussi répandu parmi les visiteurs de zoo (Baratay \& Hardouin-Fugier, 1998 : 199; Marvin, 2008). On prend ainsi le sourire du macaque rhésus pour une manifestation de joie alors qu'il signale sa soumission. Il est souvent purement métaphorique, au sens où l'attribution de propriétés humaines n'est pas prise au sérieux (Servais, 2009). En ce sens, loin de remettre en cause l'anthropocentrisme naturaliste, cet anthropomorphisme le conforte en entretenant l'idée que l'attribution d'états mentaux à des animaux captifs ne peut être qu'un jeu (ibid.). Servais (1999) montre que cet anthropocentrisme est lié au mode de présentation des animaux. En exposant l'animal en tant qu'objet offert au regard et non en tant que sujet d'actions (Hediger, 1953 : 227), les zoos présentent des animaux «étroitement contrôlés » (Servais, 1999) dont le public ne peut percevoir que difficilement l'agentivité (Warkentin, 2009).

Si l'anthropomorphisme métaphorique est parfois présent chez les soigneurs, ils peuvent néanmoins concevoir les animaux comme les sujets de leurs actions. Certes, le naturalisme est toujours présent: D. parle d'« une belle collection» et déclare que la femelle jabiru est « un bel exemplaire ». Cependant ce naturalisme semble se replier au second plan lorsqu'il s'agit d'interactions. En entrant en relation avec des animaux dont la volonté n'est pas subordonnée à la volonté humaine, D. fait l'expérience d'une véritable intersubjectivité qui l'éloigne du naturalisme (Descola, 2005: 397). La psychologie cognitive suggère que l'anthropomorphisme se manifeste essentiellement dans les interactions (Airenti, 2012). Plus précisément, l'anthropomorphisme animalocentrique est lié à des interactions marquées par l'expérience et la connaissance, le savoir et la familiarité. "On les connaît bien, y'a une vraie interaction », souligne une animalière (Estebanez, 2010). Ni «profane» ni «scientifique» (Hochadel, 2011), l'anthropomorphisme animalier est un anthropomorphisme expert (Despret, 2007). 


\section{Conclusion}

Si le parc zoologique constitue un espace où l'animal dépend de l'homme, cette dépendance ne signifie pas sa passivité. En effet, si la captivité réduit l'activité animale et peut conduire à certaines pathologies (Joulian \& Abegg, 2008), l'animal de zoo peut manifester une véritable agentivité. C'est notamment le cas lorsqu'il interagit avec des animaliers, a fortiori si ceux-ci lui ménagent des possibilités d'action. En effet, loin de toujours exercer un "contrôle direct » sur les animaux captifs, les soigneurs ont parfois l'occasion de choisir une approche favorisant les initiatives animales. Les animaliers peuvent ainsi développer des relations sociales avec des animaux dont la "volonté » acquiert une sorte de priorité. Cet anthropomorphisme zoocentrique les éloigne de l'anthropocentrisme du zoo et de la plupart de ses visiteurs. En reléguant provisoirement le naturalisme au second plan, il renforce l'agentivité des animaux captifs et donne tout son sens à la notion d'interaction. En effet, si l'agentivité peut être définie comme le " pouvoir d'un affectant sur un affecté », dans le cadre d'une interaction la distinction entre agent et patient n'est que relative. «Il ne s'agit plus [...] de l'opération d'un agent sur un objet inerte, ni de l'action en retour d'un objet, promu au rôle d'agent, sur un sujet qui se serait dépossédé en sa faveur sans rien lui demander en retour, c'est-à-dire de situations comportant, d'un côté ou de l'autre, une certaine dose de passivité : les êtres en présence s'affrontent à la fois comme des sujets et comme des objets » (Lévi-Strauss, 1962/1990 : 266).

\section{Bibliographie}

Airenti G. (2012) « Aux origines de l'anthropomorphisme. Intersubjectivité et théorie de l'esprit », Gradhiva, 15, pp. 34-53.

Altmann J. (1974) « Observational study of behavior: sampling methods », Behaviour, vol. 49, 3, pp. 227-267.

Baratay É. \& Hardouin-Fugier É. (1998), Zoos. Histoire des jardins zoologiques en Occident (XVIe-XXe siècle), Paris, La Découverte, 294 p.

Bateson G. (1936/1986) La Cérémonie du Naven, Paris, LGF, 348 p.

Berger J. (1980) «Why look at animals? », in About looking, Londres, Writers and riders, pp. 3-28.

Brunois F. (2005) «Pour une approche interactive des savoirs locaux: l'ethnoéthologie », Journal de la Société des Océanistes, 120, pp. 31-40.

Deputte B. (2007) «Comportements d'agression chez les vertébrés supérieurs, notamment chez le chien domestique (canis familiaris) », Bulletin de l'Académie Vétérinaire de France, tome 160, 5, pp. 349-358.

Descola P. (2005) Par-delà nature et culture, Paris, Gallimard, 640 p.

Descola P. (2011) L'Écologie des autres. L'anthropologie et la question de la nature, Versailles, Quae, $110 \mathrm{p}$.

Despret V. (2002) Quand le loup habitera avec l'agneau, Paris, Les Empêcheurs de penser en rond, $284 \mathrm{p}$. 
Despret V. (2007) «L'affectivité au cœur des processus de professionnalisation. Le cas des sciences du comportement animal », in F. Charvolin, A. Micoud et L. K. Nyhart (dir.), Des sciences citoyennes? La question de l'amateur dans les sciences naturalistes, la Tour d'Aigues, Éditions de l'Aube, pp. 56-73.

Despret V. (2012) Que diraient les animaux, si... on leur posait les bonnes questions?, Paris, La Découverte, 325 p.

EAZA (2008) EAZA Minimum Standards for the Accommodation and Care of Animals in Zoos and Aquaria.

http://www.eaza.net/about/Documents/Standards_2008.pdf (consulté le 26/09/12).

Estebanez J. (2010) « Ceux qui sont proches: les soigneurs au zoo », Sociétés, 108, pp. 47-57.

Estebanez J. \& Staszak J.-F. (2012) «Animaux humains et non-humains au zoo. L'expérience de la frontière animale », in D. Dubied, D. Gerber D. et J. J. Fall (dir.), Aux frontières de l'animal. Mises en scène et réflexivité, Genève, Droz, pp. 149-174.

Ferret C. (2006), Techniques iakoutes aux confins de la civilisation altaïque du cheval. Contribution à une anthropologie de l'action, thèse de doctorat, Paris, EHESS, 1239 p.

Ferret C. (2012) «Vers une anthropologie de l'action. André-Georges Haudricourt et l'efficacité technique », L'Homme, 202, pp. 113-139.

Fontaine L. (2010) " "Agents" ou "patients"? De l'agentivité des chamanes yucuna d'Amazonie colombienne », Ateliers du LESC, 34

http://ateliers.revues.org/8526(consulté le 12/09/12).

Gibson J. J. (1979) The Ecological Approach to Visual Perception, Boston, Houghton Mifflin, $332 \mathrm{p}$.

Goldberg J. (1998) Les Sociétés animales, Lausanne, Paris, Delachaux et Niestlé, 345 p.

Goodall J. (1986) The Chimpanzees of Gombe. Patterns of Behavior, Cambridge, Belknap Press of Harvard University Press, 674 p.

Hediger H. (1942/1953), Les Animaux sauvages en captivité. Introduction à la biologie des jardins zoologiques, Paris, Payot, 247 p.

Hochadel O. (2011) «Watching exotic animals next door: "scientific" observations at the zoo (ca. 1870-1910) », Science in Context, 24/2, pp. 183-214.

Hoyo J. et al. (1992) Handbook of the Birds of the World, vol. 1., Barcelona, Lynx Edicions, 696 p.

Joulian F. (1999) «Observer des primates dans la nature. Réflexions anthropologiques autour de l'habituation », Gradhiva, n ${ }^{\circ} 25$, pp. 79-91.

Joulian F. \& Abegg C. (2008) «Zoos et cause animale. Perspectives éthologique et anthropologique », Techniques \& Culture, $\mathrm{n}^{\circ}$ 50, pp. 120-143.

Karlsson F. (2012) «Critical anthropomorphism and animal ethics », Journal of Agricultural and Environmental Ethics, 25, 5, pp. 707-720.

Latour B. (1991), Nous n'avons jamais été modernes. Essai d'anthropologie symétrique, Paris, La Découverte, 210 p.

Leblan V. (2011) «Les rendez-vous manqués de l'ethnologie et de la primatologie de terrain (1960-2010) », Revue de primatologie, 3

http://primatologie.revues.org/808

(Consulté le 20 septembre 2012).

Lestel D., Brunois F., et Gaunet F. (2006) «Etho-ethnology and ethno-ethology », Social Science Information, 45/2, pp. 155-177. 
Lévi-Strauss C. (1962/1990) La Pensée sauvage, Paris, Presses Pocket, 347 p.

Maher C. R. \& Lott D. F. (1995) «Definitions of territoriality used in the study of variation in vertebrate spacing systems ", Animal Behaviour, 49/6, pp. 1581-1597.

Marvin G. (2008) «L'animal de zoo. Un rôle entre sauvage et domestique », Techniques \& Culture, 50, pp. 102-119.

Merleau-Ponty M. (1995) La Nature. Notes et cours du Collège de France, Paris, Éditions du Seuil, 374 p.

Mullan B. \& Marvin G. (1999) Zoo Culture, Urbana, Chicago, University of Illinois Press, 208 p.

Nakamura M. (2009) «Interaction studies in Japanese primatology. Their scope, uniqueness, and the future », Primates, 50/2, pp. 142-152.

Pellegrini P. (1995) «Zoos, parcs et réserves, quel est le statut de ces animaux offerts au regard de l'homme ? », in B. Lizet et G. Ravis-Giordiani (dir.), Des bêtes et des hommes. Le rapport à l'animal : un jeu sur la distance, Paris, CTHS, pp. 227-242.

Porcher J. (2003) «Bien-être et souffrance en élevage : conditions de vie au travail des personnes et des animaux », Sociologie du travail, 45, pp. 27-43.

Rémy É. (2007) «Familiarité et détachement envers l'animal: des ajustements inattendus. Le cas de la loutre dans la nature et dans les zoos ", in F. Charvolin, A. Micoud et L. K. Nyhart (dir.), Des sciences citoyennes? La question de l'amateur dans les sciences naturalistes, la Tour d'Aigues, Editions de l'Aube, pp. 40-55.

Rivas J. A. \& Burghardt G. M. (2002) «Crotalomorphism: A metaphor for understanding anthropomorphism by omission », in M. Bekoff, C. Allen, \& G. M. Burghardt (dir.), The Cognitive Animal: Experimental and Theoretical Perspectives on Animal Cognition, Cambridge, MA, MIT Press, pp. 9-17.

Servais V. (1999) «Zoos, éducation et malentendus. Essai d'anthropologie des émotions du visiteur de zoo », Cahiers d'Éthologie, 19/1, pp. 1-16.

Servais V. (2009) «"Dialogues" avec les singes : l'anthropomorphisme comme mode de relation dans les rencontres entre visiteurs et primates en zoo » http://hdl.handle.net/2268/26341 (consulté le 18/07/12)

Servais C. \& Servais V. (2009) « Le malentendu comme structure de la communication », Questions de communication, 15, pp. 21-49.

Simmel G. (1908/1999) Sociologie. Étude sur les formes de la socialisation, Paris, PUF, $772 \mathrm{p}$.

Uexküll J. von (1934/1965) Mondes animaux et monde humain suivi de Théorie de la signification, Paris, Denoël, 188 p.

Vialles N. (2004) «La nostalgie des corps perdus », in F. Héritier et M. Xanthakou (dir.), Corps et affects, Paris, Odile Jacob, pp. 275-289.

Waal F. de (1999) "Anthropomorphism and anthropodenial. Consistency in our thinking about humans and other animals », Philosophical Topics, vol. 27, n 1 , pp. 255280 .

Warkentin T. (2009) «Whale agency: affordances and acts of resistance in captive environments ", in S. E. McFarland \& R. Hediger (dir.), Animals and Agency. An Interdisciplinary Exploration, Leiden, Koninklijke Brill NV, pp. 23-43. 